\title{
NUMERICAL STUDY OF FLOW OF A NON-NEWTONIAN FLUID OVER A ROTATING CYLINDER
}

\author{
Nguyen Thi Hong Nhung ${ }^{1}$, Pham Thanh Huyen ${ }^{2}$, Nguyen Cong Vinh ${ }^{1}$, Bui Mai Cuong ${ }^{*}$ \\ ${ }^{1}$ The University of Danang - University of Technology and Education, \\ ${ }^{2}$ Hochiminh City University of Technology and Education
}

\begin{tabular}{|c|c|}
\hline ARTICLE INFO & \multirow{12}{*}{$\begin{array}{l}\text { In this work, flow characteristics of a non-Newtonian fluid exhibiting } \\
\text { yield stress property over a rotating unconfined two-dimensional (2D) } \\
\text { cylinder under a wide range of rotational speed }(\alpha=0-10) \text { are studied } \\
\text { using the Computational Fluid Dynamics (CFD) approach. The fluid } \\
\text { considered is clay suspension with a kaolinite mass concentration of } \\
15 \mathrm{wt} \% \text {. To describe complex rheological behaviors, the Herschel- } \\
\text { Bulkley model is utilized. Various flow aspects at Reynolds number } \\
(\mathrm{Re}) \text { of } \mathrm{Re}=50-500 \text {, i.e., streamlines, development of solid-like and } \\
\text { flowing regions, and hydrodynamic forces are reported and analyzed. } \\
\text { Results show that the near-field rigid zones only form with } \alpha \leq 1 \text {. } \\
\text { Moreover, the flowing region becomes smaller at Re }=50 \text { but tends to } \\
\text { enlarge at } \operatorname{Re}=100 \text { and } 500 \text { with the increasing } \alpha \text {. Furthermore, the } \\
\text { estimated forces acting on the cylinder are found to be greatly } \\
\text { dependent on both Re and } \alpha \text {. }\end{array}$} \\
\hline Received: $19 / 7 / 2021$ & \\
\hline Revised: 08/11/2021 & \\
\hline & \\
\hline & \\
\hline KEYWORDS & \\
\hline Non-Newtonian fluid & \\
\hline Yield stress & \\
\hline CFD & \\
\hline & \\
\hline aolinite-water mixture & \\
\hline Herschel-Bulkley's model & \\
\hline
\end{tabular}

\section{NGHIÊN CÚU ĐẶC TÍNH DÒNG CHẢY LUUU CHẤT PHI NEWTON QUA TRỤ TRÒN XOAY BĂNG PHƯƠNG PHÁP MÔ PHỎNG SỐ}

\author{
Nguyễn Thị Hồng Nhung ${ }^{1}$, Phạm Thanh Huyền ${ }^{2}$, Nguyễn Công Vinh ${ }^{1}$, Bùi Mai Cường ${ }^{1 *}$ \\ ${ }^{1}$ Truòng Đại học Su phạm Kỹ thuật - ĐH Đà Nẵng \\ ${ }^{2}$ Truò̀ng Đại học Su phạm Kỹ thuật Thành phố Hồ Chí Minh
}

\begin{tabular}{|c|c|}
\hline THÔNG TIN BÀI BÁO & TÓM TẮT \\
\hline Ngày nhận bài: 19/7/2021 & \multirow{11}{*}{ 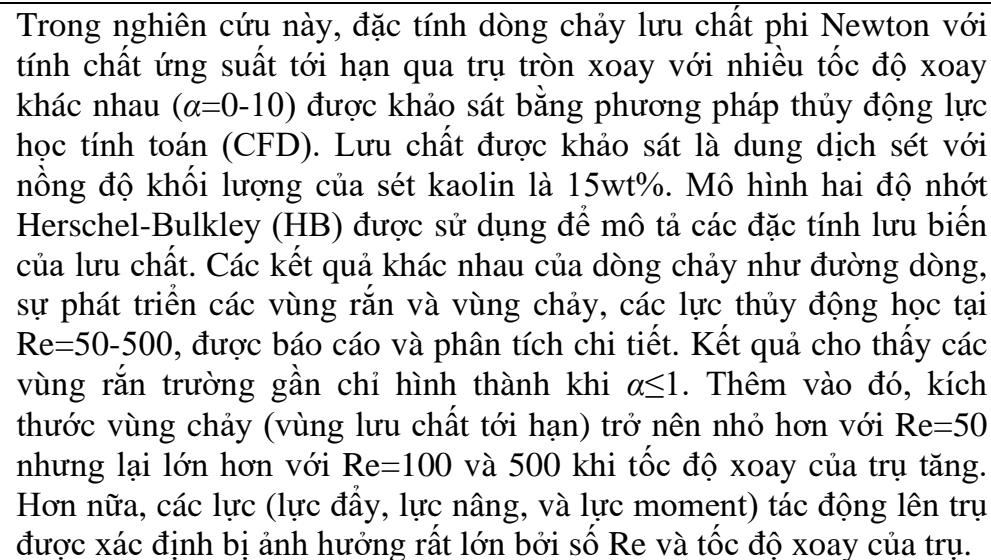 } \\
\hline Ngày hoàn thiện: $08 / 11 / 2021$ & \\
\hline & \\
\hline Ngày đăng: 09/11/2021 & \\
\hline & \\
\hline TÙ' KHÓA & \\
\hline Lưu chất phi Newton & \\
\hline Úng suất tới hạn & \\
\hline Phương pháp CFD & \\
\hline Dung dịch sét kaolin & \\
\hline Mô hình Herschel-Bulkley & \\
\hline
\end{tabular}

DOI: $\underline{\text { https://doi.org/10.34238/tnu-jst.4785 }}$

* Corresponding author. Email: bmcuong@ute.udn.vn 


\section{Introduction}

Despite the fact that non-Newtonian (fluid) materials are frequently found in nature (e.g., lava, debris flows, and melting permafrost ice [1]-[3]) and many engineering flows (e.g., polymer, fresh concrete, or crude oil [4]-[6]), their hydrodynamic behaviors are still poorly understood. The deformation of these materials does not obey Newton's law of viscosity, i.e. their viscosity can be significantly varied during the flowing process due to rheologically complex properties such as shear-dependence and yield stress. For those possessing the latter, their flowing occurs only when the applied shear stress is greater than the yield stress of the materials; otherwise, they are considered solid. With this, solid-like regions, in which the fluid was unyielded, can be formed far-field covering sheared zone (i.e., far-field unyielded zone) and scatteredly built in the flow field pattern (i.e., moving rigid zone) and/or attach to obstacles' surface (i.e., static rigid zone). It is obvious that the flow characteristics of such fluid are very different and much complex compared to those of a Newtonian one. As can be seen in Fig. 1, the yield stress effect seems to stabilize the flow and leads to the development of rigid zones.

As a classical problem of fluid mechanics, the flow over a stationary cylinder has been well studied for the Newtonian liquids. For the non-Newtonian ones, Tokpavi et al. investigated the creeping flow of viscoplastic Bingham [7] and Herschel-Bulkley [8] fluids at very high Oldroyd (Od) numbers. Results showed that yield stress characteristics had a strong impact on the formation of unyielded zones and the acting forces on the cylinder. Moreover, at higher Re, Mossaz et al. [9] numerically and experimentally characterized the flow morphology of Carbopol gel exhibiting both shear-thinning and yield stress properties. Various aspects, i.e., flow field pattern, formation of rigid zones, and characteristic lengths of circulation wake, were revealed. Recently, Syrakos et al. [10] carried out simulations to study the microstructural evolution mechanism of a thixotropic fluid flow over a cylinder. Thixotropic effects were then observed to considerably affect the hydrodynamic behaviors of a yield stress liquid.

The works on complex fluids over a rotating cylinder are much fewer; additionally, a numerical approach was mainly employed. Townsend [11] and then Panda and Chhabra [12] found that the flow behaviors, and drag and lift coefficients were strongly dependent on such rheological characteristics as viscoelasticity or shear-thinning and dimensionless rotational rate $\alpha$. Additionally, Thakur et al. [13] performed a rigorous numerical study on a Bingham fluid with $\alpha=0-6, \mathrm{Re}=0.1-40$ and $\mathrm{Bn}=0-1000$. Various results for flow streamlines and unyielded/yielded zones were reported in detail.

It is noted that the fluid used in most of the aforementioned works was artificial. Our work is aiming at investigating complex behaviors of real yield stress fluid, i.e., kaolin clay suspension, and the influences of rotational motion on the flow of this rheological liquid. It is good to mention that this setting has numerous practical applications such as in fluids mixing, coating or oil drilling processes.

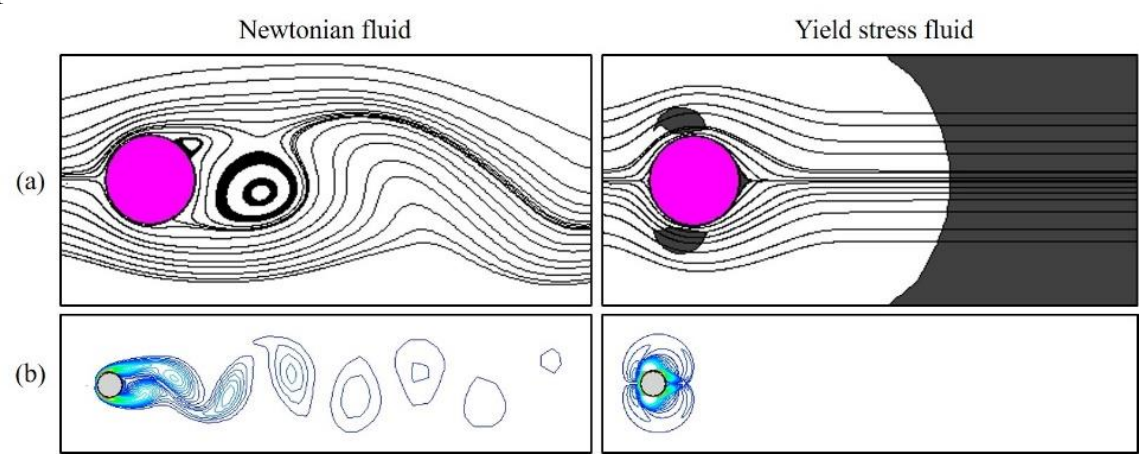

Figure 1. Comparison in (a) flow field pattern and (b) vorticity distribution between Newtonian (water) and non-Newtonian (kaolinite-water mixture) fluids at $R e=100$. Shaded areas represent solid-like regions 


\section{Methodology}

\subsection{Governing equations}

Mass and momentum conservation equations are as follows,

$$
\begin{gathered}
\nabla \cdot \underline{u}=0, \\
\rho \underline{u} \cdot \nabla \underline{u}=\rho \underline{f}+\nabla \cdot \underline{\sigma} .
\end{gathered}
$$

Here, $\underline{u}$ and $f$ are the velocity and body force vectors, respectively; $\rho$ is the fluid density; and $\underline{\underline{\sigma}}=-p \underline{I}+\underline{\underline{\tau}}$ is the total stress tensor with $p$ being the pressure, $\underline{I}$ the unit tensor, $\underline{\underline{\tau}}=2 \mu \underline{\dot{\gamma}}$ the deviatoric stress tensor, and $\underset{\gamma}{\dot{\gamma}}$ the deformation rate tensor.

\subsection{Modeling of yield stress behaviors}

To express yield stress characteristics, the well-known bi-viscosity Herschel-Bulkley (HB) model is employed as:

$$
\left\{\begin{array}{ll}
\underline{\underline{\tau}}=\left(K+\frac{\tau_{0}}{\dot{\gamma}}\right) \underline{\dot{\gamma}} & \text { if } \tau>\tau_{0} \\
\dot{\gamma}=0 & \text { if } \tau \leq \tau_{0}
\end{array},\right.
$$

with $\tau$ the intensity of extra-stress; $\tau_{0}$ the yield stress; $K$ the plastic viscosity; and $\dot{\gamma}$ the strain rate tensor's magnitude. In Ansys Fluent, the HB model is modified as:

$$
\left\{\begin{array}{ll}
\mu=K+\frac{\tau_{0}}{\dot{\gamma}} & \text { if } \dot{\gamma}>\dot{\gamma}_{c} \\
\mu=\frac{\tau_{0}\left(2-\frac{\gamma}{\gamma_{c}}\right)}{\gamma_{c}}+K\left(1+\frac{\gamma}{\gamma_{c}}\right) & \text { if } \dot{\gamma} \leq \dot{\gamma}_{c}
\end{array}\right. \text {. }
$$

Here, $\dot{\gamma}_{c}$ is the deformation rate at the yield point of $\tau=\tau_{0}$. It is noteworthy that the fluids used in our work for Newtonian and non-Newtonian solutions are, respectively, water and kaolinitewater mixture with the kaolinite mass fraction of $15 \mathrm{wt} \%$. Rheological properties of the latter, i.e., the yield stress of $\tau_{0}=0.8 \mathrm{~Pa}$, the apparent viscosity of 1.18 , and the critical shear rate of $\dot{\gamma}_{c}=0.01$ $1 / \mathrm{s}$, can be referred to Lin et al. [14] Moreover, the unyielded (solid-like) regions are defined by $\tau \leq \tau_{0}$ as in [15].

\subsection{Non-dimensional numbers}

The flow is characterized by Reynolds number $(\mathrm{Re})$ as:

$$
\operatorname{Re}=\frac{\rho u_{0} D}{K},
$$

with $u_{0}$ the far-field velocity and $D$ the cylinder's diameter. Non-dimensional rotational speed is defined as:

$$
\alpha=\frac{\omega D}{2 u_{0}},
$$

with $\omega$ the angular speed.

\subsection{Computational implementation}

A two-dimensional (2D) circular domain with the diameter of $D_{d}=200 D$ is created (see Fig. 2). For boundary conditions, inlet velocity $u_{0}$ and outlet pressure $p=0$ is, respectively, applied for 
the front half and rear half of the domain. Moreover, the (rotational) moving condition is used for the cylinder's wall; note that counter-clockwise is chosen as the positive direction.

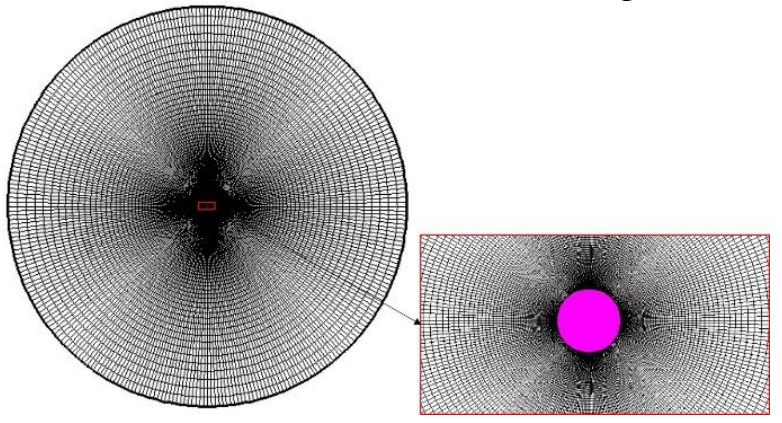

Figure 2. Domain and mesh

A structured mesh with high near-field refinement is generated for calculations (see Fig. 2). In order to determine a suitable resolution, a mesh convergence study is carried out. Figure 3 shows a comparison in yield boundary, which separates far-field unyielded (solid) and yielded (fluid) one, among three grid resolutions including M1 (35000 elements), M2 (42000 elements), and M3 (55300 elements). As can be seen, the yield line is almost converged with M2. Furthermore, it is observed that three meshes provide identical results for drag coefficient $\left(C_{d}=16.41\right)$ but M3 costs significantly more computational time. Mesh resolution M2 is obviously the most optimal and therefore employed for all simulations.

A Finite Volume Method (FVM)-based commercial package named Ansys Fluent is employed to solve the problem. Moreover, second-order schemes are used for spatial discretization.

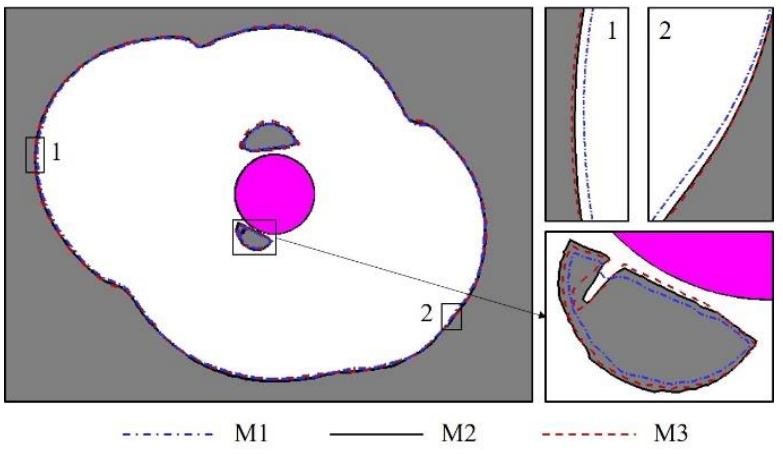

Figure 3. Mesh convergence study: variation in yield boundary with various mesh resolution with $\alpha=1$ at $R e=100$

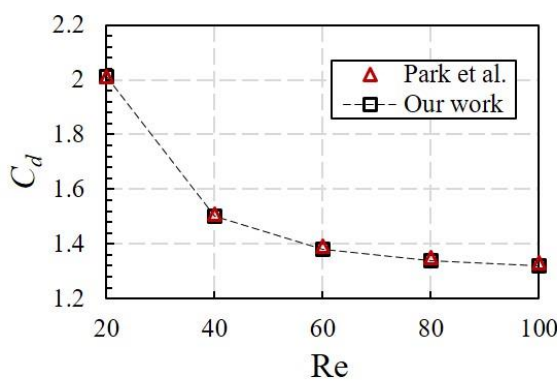

Figure 4. $C_{d}$ as a function of Re: Our results for a Newtonian fluid vs. Park et al. 's [16]

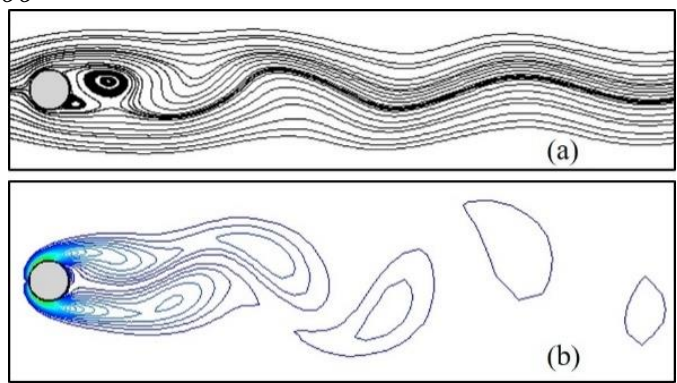

Figure 5. Results for (a) flow streamlines and (b) vorticity field of a Newtonian liquid at Re=60

For validation purposes, results of Newtonian fluid flows produced by our numerical strategy are compared with available data in the literature. As can be seen in Fig. 4, our results for drag force have a perfect agreement with those of Park et al. [16]. Furthermore, it is good to be noticed 
that our simulated flow morphology at $\mathrm{Re}=60$ also matches well with experimental results in [17] (see Fig. 5). Specifically, both works indicate that the flow at this Re is in a non-stationary regime with vortex manifesting downstream.

\section{Results and Discussion}

\subsection{Flow morphology}

In this part, simulated results for the flow field of kaolinite suspension $15 \mathrm{wt} \%$, a typical yield stress liquid, under a wide range of rotational speed $(0 \leq \alpha \leq 10)$ are presented. Reynolds number is varied in $\mathrm{Re}=50-500$.
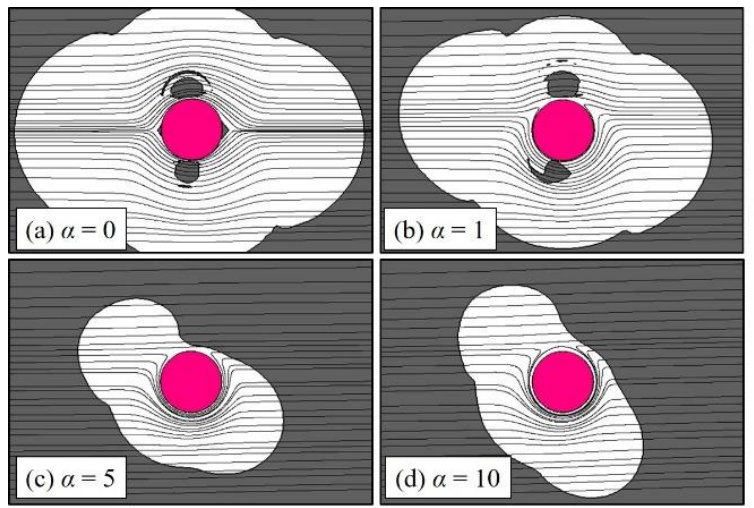

(e) Yield boundary

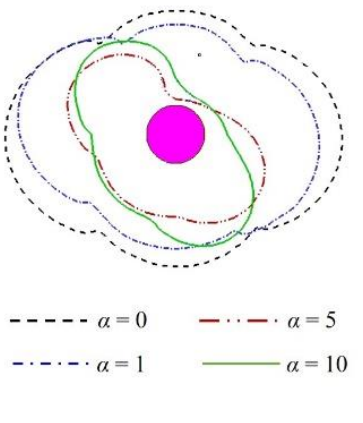

Figure 6. Flow streamlines and solid-like regions (shaded) of a yield stress fluid (Kaolinite suspension 15 wt\%) with (a) $\alpha=0$, (b) $\alpha=1$, (c) $\alpha=5$ and (d) $\alpha=10$ at $R e=50$. Variations in yield boundary with various $\alpha$ are also presented (e)

Figure 6 illustrates results for flow morphology at $\mathrm{Re}=50$ with various rotational rates. When $\alpha=0$, it is seen that the yield stress flow is still in a creeping regime without any downstream recirculation wakes or vortex shedding meanwhile at this Re, the Newtonian fluid flow begins to enter an unsteady mode as claimed in [18]. This can be attributed to the appearance of solid-like regions impeding the fluid flowing. Indeed, when the cylinder is stationary, there exist two nearfield static rigid zones at stagnation points and two moving ones at its upper and lower parts. This finding is in line with Tokpavi et al. [7], [8] for the very slow yield stress fluid within which the viscoplastic effect is predominant. The moving rigid zones still exist when $\alpha=1$; however, with high $\alpha$, e.g., $\alpha=5$ or 10 , no near-field rigid but only a large far-field unyielded zone is formed. Furthermore, the flowing region (inside the yield boundary) is surprisingly found to narrow with the increasing $\alpha$, resulting in the reduction of material mixing efficiency at the near-field of the cylinder (see Fig. 6e).
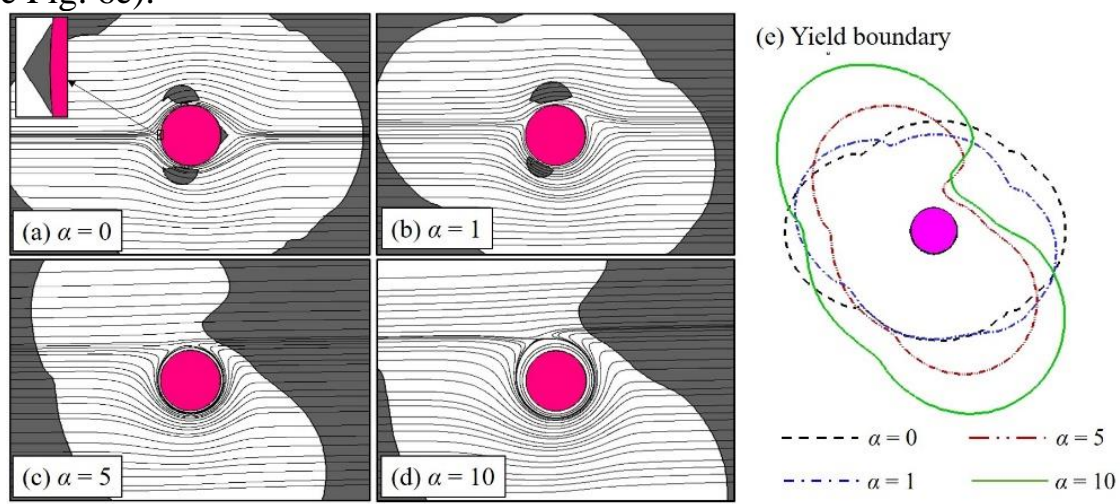

Figure 7. Flow streamlines and solid-like regions (shaded) of a yield stress fluid (kaolinite suspension $15 w t \%$ ) with (a) $\alpha=0$, (b) $\alpha=0$, (c) $\alpha=0$ and (d) $\alpha=10$ at Re=100. Variations in yield boundary with various $\alpha$ are also presented (e) 
Results for flow morphology at $\mathrm{Re}=100$ with different values of $\alpha$ are presented in Fig. 7. Compared to the case of $\mathrm{Re}=50$, the distribution of near-field solid-like regions is relatively similar though they differ in shape and size. In detail, static and moving rigid zones are, respectively, detected with $\alpha=0$ and $\alpha \leq 1$. However, the yield boundary at $\operatorname{Re}=100$ is observed to slightly enlarge as $\alpha$ increases; this finding is opposite to the results at $\mathrm{Re}=50$ (see Fig. 7e).

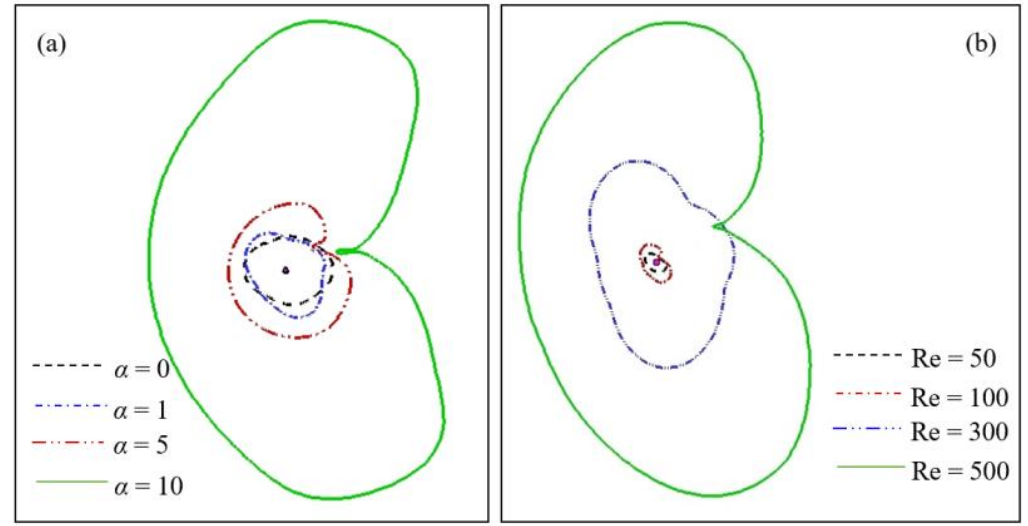

Figure 8. Variation in yield boundary with various $\alpha$ at $R e=100$ (a) and at various Re with $\alpha=5$ (b)

The effect of the rotational speed on the size of the yield boundary seems to be much more pronounced at $\mathrm{Re}=500$. As can be seen in Fig. 8a, the yielded region gets drastically greater as $\alpha$ increases. Moreover, the influences of the inertial effect described by Reynolds numbers on the formation of the yielded region are also investigated. Figure $8 \mathrm{~b}$ shows the results for the yield boundary at Re ranged from 50 to 500. It is evident that the higher Re, the considerably greater the flowing zones are formed, leading to the incredible improvement of the materials mixing process.

\subsection{Hydrodynamic forces}

Results for drag, lift and torque forces are reported are report in this part. The drag, lift and moment coefficients are, respectively, defined as:

$$
\begin{aligned}
C_{d} & =\frac{F_{d}}{2 \rho u_{0}^{2} D}, \\
C_{d} & =\frac{F_{d}}{2 \rho u_{0}^{2} D}, \\
C_{m} & =\frac{M}{0.5 u_{0}^{2} A L} .
\end{aligned}
$$

Here, $F_{d}, F_{l}$, and $M$ stand for the drag force, lift force, and moment about the $z$-axis, respectively. Moreover, $A$ and $L$ are, in turn, the reference area and the length of the cylinder.

Figure 9 reveals results for $C_{d}$ with various values of rotational speed at $\mathrm{Re}=50,100$ and 500 . As can be observed, an increase in Re results in a sharp increase in estimated $C_{d}$ at the same $\alpha$; nevertheless, the deviation is seen to be reduced when $\alpha$ increases. For instance, when the cylinder does not move, $C_{d}$ provided at $\mathrm{Re}=50$ is 40 times greater than that of $\mathrm{Re}=500$ meanwhile the difference is only of 7.4 times with $\alpha=10$. Moreover, each case of Re studied shows a different correlation between $C_{d}$ and $\alpha$. For instance, at $\mathrm{Re}=50, C_{d}$ gets smaller with the increasing $\alpha$. This is, however, not the case for $\mathrm{Re}=100$ and 500; in detail, at $\mathrm{Re}=100, C_{d}$ decreases to the minimum value at $C_{d}=11.14$ at $\alpha=5$ and then increases as $\alpha$ rises to 10 . It is even more complicated with $\mathrm{Re}=500$ in which an up-and-down $C_{d}$ - $\alpha$ curve is seen; experimental works should be required to confirm the phenomena and find out the mechanism for this. Furthermore, results for lift and moment coefficients with different $\alpha$ are presented in Fig. 10. Generally, the larger rotational speed, the greater magnitudes of $C_{l}$ and $C_{m}$ are found. 


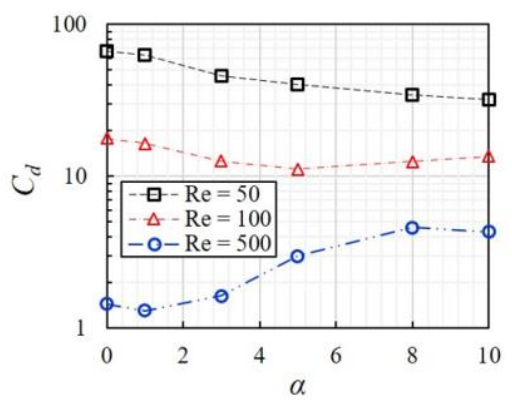

Figure 9. $C_{d}$ as a function of rotational rate $\alpha$
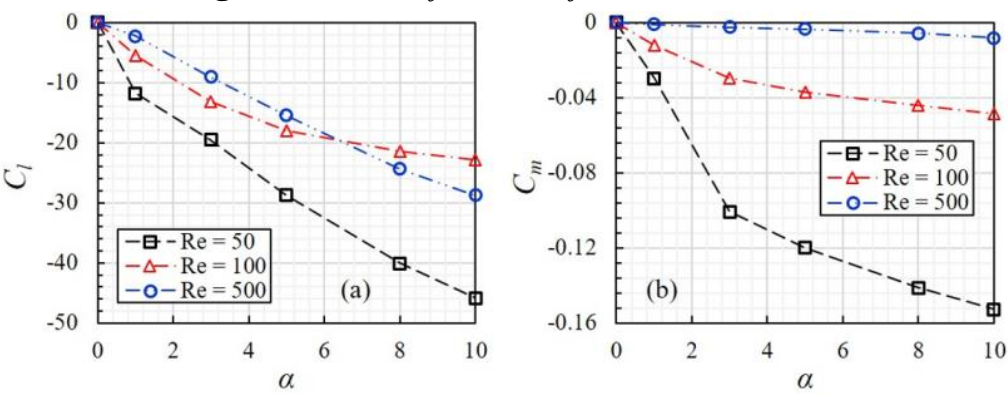

Figure 10. (a) $C_{l}$ and (b) $C_{m}$ as a function of rotional rate $\alpha$

\section{Conclusion}

An investigation on a real yield stress fluid flow over a rotating cylinder is carried out using a numerical approach. In detail, various hydrodynamic behaviors of clay suspension with $15 \mathrm{wt} \%$ kaolinite were studied at Re ranged in $\mathrm{Re}=50-500$ and with the rotational rate of $\alpha=0-10$.

At $\operatorname{Re}=50$, when the rotational movement was not applied, the viscoplastic fluid flow was revealed to be still creeping; this was very different from a Newtonian one whose regime was nonstationary with the appearance of downstream von-Karman streets. Moreover, the near-field solidlike regions, which were supposed to block the flowability, were observed to be formed when $\alpha \leq 1$. Furthermore, when $\alpha$ became higher, the flowing zone was seen to be smaller at this Re.

At $R e=100$, the solid-like regions were found to be placed in similar positions with $R e=50$ but had significant changes in their shape and size. More interestingly, opposite to the case of $\operatorname{Re}=50$, there existed a considerable enlargement in the yielded region for $\mathrm{Re}=100$ and 500. Additionally, the increase in Re could also result in the dramatic expansion of this region, thereby significantly improving the fluids mixing.

In addition, the drag coefficient $C_{d}$ was found to strongly depend on both $\operatorname{Re}$ and $\alpha$. In detail, as Re increased, $C_{d}$ considerably decreased. Moreover, $C_{d}$ reduced with the increasing $\alpha$ at $\mathrm{Re}=50$; however, the $C_{d^{-}} \alpha$ correlation became complex at $\mathrm{Re}=100$ and 500, more studies should be performed to discover the mechanism of these cases. Furthermore, in general, when $\alpha$ got higher, the magnitudes of $C_{l}$ and $C_{m}$ were noted to be generally greater.

In the future, three-dimensional (3D) simulations would be conducted. Moreover, we also plan to investigate the yield stress behaviors in a transition and/or turbulent flow regimes.

\section{REFERENCES}

[1] A. J. Harris and S. K. Rowland, "Lava flows and rheology" in The encyclopedia of volcanoes, H. Sigurdsson, B. Houghton, S. McNuut, H. Rymer and J. Stix, Eds., 2nd ed. Elsevier, 2015.

[2] A. Scotto di Santolo, A. M. Pellegrino, and A. Evangelista, "Experimental study on the rheological behaviour of debris flow," Natural Hazards and Earth System Sciences, vol. 10, no. 12, pp. 2507 2514, 2010. 
[3] W. F. Budd and T. Jacka, "A review of ice rheology for ice sheet modelling," Cold Regions Science and Technology, vol. 16, no. 2, pp. 107-144, 1989.

[4] S. M. Kamal, A. S. Sultan, U. A. Al-Mubaiyedh, and I. A. Hussein, "Review on polymer flooding: rheology, adsorption, stability, and field applications of various polymer systems," Polymer Reviews, vol. 55, no. 3, pp. 491-530, 2015.

[5] N. Roussel, "Rheology of fresh concrete: from measurements to predictions of casting processes," Materials and Structures, vol. 40, no. 10, pp. 1001-1012, 2007.

[6] X. Xin, Y. Li, G. Yu, W. Wang, Z. Zhang, M. Zhang, W. Ke, D. Kong, K. Wu, and Z. Chen, "NonNewtonian characteristics of heavy oil in the Bohai bay oil field: Experimental and simulation studies," Energies, vol. 10, no. 11, p. 1698, 2017.

[7] D. L. Tokpavi, A. Magnin, and P. Jay, "Very slow flow of Bingham viscoplastic fluid around a circular cylinder," Journal of Non-Newtonian Fluid Mechanics, vol. 154, no. 1, pp. 65-76. 2008.

[8] D. L. Tokpavi, P. Jay, A. Magnin, and L. Jossic, "Experimental study of the very slow flow of a yield stress fluid around a circular cylinder," Journal of Non-Newtonian Fluid Mechanics, vol. 164, no. 13, pp. 35-44, 2009.

[9] S. Mossaz, P. Jay, and A. Magnin, "Experimental study of stationary inertial flows of a yield-stress fluid around a cylinder," Journal of Non-Newtonian Fluid Mechanics, vol. 189, pp. 40-52, 2012.

[10] A. Syrakos, G. C. Georgiou, and A. N. Alexandrou, "Thixotropic flow past a cylinder," Journal of Non-Newtonian Fluid Mechanics, vol. 220, pp. 44-56, 2015.

[11] P. Townsend, "A numerical simulation of Newtonian and visco-elastic flow past stationary and rotating cylinders," Journal of Non-Newtonian Fluid Mechanics, vol. 6, no. 3-4, pp. 219-243, 1980.

[12] S. K. Panda, and K. P. Chhabra, "Laminar flow of power-law fluids past a rotating cylinder," Journal of Non-Newtonian Fluids Mechanics, vol. 165, no. 21-22, pp. 1442-1461, 2010.

[13] P. Thakur, S. Mittal, N. Tiwari, and R. P. Chhabra, "The motion of a rotating circular cylinder in a stream of Bingham plastic fluid," Journal of Non-Newtonian Fluid Mechanics, vol. 235, pp. 29-46, 2016.

[14] Y. Lin, N. Phan-Thien, J. B. P. Lee and B. C. Khoo, "Concentration dependence of yield stress and dynamic moduli of kaolinite suspensions," Langmuir, vol. 31, no. 16, pp. 4791-4797, 2015.

[15] G. R. Burgos, A. N. Alexandrou, and V. Entov, "On the determination of yield surfaces in HerschelBulkley fluids," Journal of Rheology, vol. 43, no. 3, pp. 463-483, 1999.

[16] J. Park, K. Kwon, and H. Choi, "Numerical solutions of flow past a circular cylinder at Reynolds numbers up to 160,” KSME International Journal, vol. 12, no. 6, pp. 1200-1205, 1998.

[17] D. J. Tritton, "Experiments on the flow past a circular cylinder at low Reynolds numbers," Journal of Fluid Mechanics, vol. 6, no. 4, pp. 547-567, 1959.

[18] C. H. Williamson, "Vortex dynamics in the cylinder wake," Annual review of fluid mechanics, vol. 28, no. 1, pp. 477-539, 1996. 